\title{
Entre nationalisme, réactivations et pacifisme : La Première Guerre mondiale inspiratrice de chansons. Perspectives franco-allemandes (1915-2014)
}

\section{Gilles Buscot}

Université de Strasbourg

\begin{abstract}
Sowohl während als auch nach dem "Großen Krieg" ist das Lied eine der populären künstlerischen Sprachen, die jener Konflikt hervorbrachte. Tatsächlich ist der Erste Weltkrieg auch heute noch eine Quelle der Inspiration für einige französische und deutsche Sänger. Der Artikel untersucht, wie sich dieses Verhältnis seit dem Krieg, als patriotische Lieder mit den selteneren pazifistischen Liedern konkurrierten, verändert hat. Er zeigt auf, wie einige Lieder in späteren Konfliktzeiten "reaktiviert" wurden und wie in innen der Wunsch nach Zeugenschaft, Anprangerung und Versöhnung allmählich durchgesetzt hat wenn auch in unterschiedlicher Weise auf beiden Seiten des Rheins.
\end{abstract}

Que ce soit pendant ou après la « Grande Guerre », la chanson fait partie des langages artistiques populaires que ce conflit majeur a suscités. De fait, la Première Guerre mondiale est aujourd'hui encore une source d'inspiration pour certains chanteurs français et allemands. L'article examine la manière dont le propos a changé depuis la guerre elle-même où les chants patriotiques rivalisaient avec quelques chansons pacifistes. Puis comment certaines chansons se sont « réactivées » durant d'autres périodes de conflits. Et enfin comment une volonté de témoignage, de dénonciation et de réconciliation s'est progressivement imposée à travers la chanson, mais avec des modalités différentes de part et d'autre du Rhin.

Both during or after the «Great War», song belongs to the popular artistic languages that this major conflict generated. In fact, World War I is still a source of inspiration for some French and German singers today. The article examines how this relation has changed after the war itself when patriotic songs competed with a few pacifist songs. It shows how some songs were "reactivated" during other periods of conflict, and finally how, through such songs, a desire for witness, denunciation, and reconciliation gradually took hold, but in different ways on both sides of the Rhine.

Keywords

Lied • Erster Weltkrieg • Schützengräben • Patriotismus • Pazifismus

Chanson $\cdot$ Première Guerre mondiale $\cdot$ tranchées $\cdot$ patriotisme $\cdot$ pacifisme

Song $\cdot$ World War I $\cdot$ trenches $\cdot$ patriotism $\bullet$ pacifism

Pendant et après ce conflit majeur que l'usage désigne par « la Grande Guerre », les chansons font partie des nombreux langages artistiques qu'il a suscités ou réactivés, comme autant de petites cicatrices de la catastrophe originelle du $X^{\mathrm{e}}$ siècle. La spécificité et l'intérêt de cette forme d'art tient, en l'occurrence, à son caractère hybride et populaire : hybride, car elle combine la création littéraire et musicale ; populaire, car sa brièveté et sa simplicité (tout au moins apparente) lui permettent de toucher aisément chaque citoyen et/ou chaque soldat (Saka 2004). À la différence de la « poésie pure », le texte et la mélodie d'une chanson ont en effet pour vocation de s'imprimer très vite dans l'oreille de l'auditeur, ne serait-ce que par le retour régulier et parfois obsédant d'un refrain que chacun peut fredonner à son tour, devenant co-créateur des chansons qu'il chante et qu'il " réincarne ». On comprend dès lors que les chansons, comme les affiches, ont constitué un outil de propagande particulièrement mobile et efficace durant la Première Guerre, relayé par l'industrie naissante du disque microsillon.

Comme les affiches, les chansons sur la guerre furent souvent, dans un premier temps, des œuvres de commande au service du nationalisme guerrier. Mais assez rapidement, 
à l'instar de la peinture par exemple, on peut parler d'une rivalité entre un langage artistique belligérant et celui, plus subversif, de chansons qui deviennent de plus en plus dénonciatrices et pacifistes au fil du temps. Sur la base de quelques exemples emblématiques, on examinera ici comment le propos a changé pendant et a fortiori depuis la Première Guerre elle-même ${ }^{1}$; et dans quelle mesure, après 1918, cette guerre a été régulièrement réactivée en chansons, notamment durant d'autres périodes de menaces conflictuelles. On constatera, en définitive, que cette guerre constitue aujourd'hui encore une source d'inspiration pour certains chanteurs français pour lesquels on peut parler d'une volonté de témoignage, de dénonciation et de réconciliation alors qu'en Allemagne, la Seconde Guerre a beaucoup plus recouvert les traces chantées de la Première que ce n'est le cas en France.

\section{ENTRE NATIONALISME, NOSTALGIE DE LA PERTE ET DÉNONCIATION}

\section{Nostalgie de la perte et appel au combat}

Les chansons nationalistes et martiales se fondent souvent sur une nostalgie de ce que la nation a perdu ou risque de perdre à cause de la guerre. II s'agit de se situer en position de victime, et, partant, d'appeler au sursaut armé, de plaider la légitime défense face au pays voisin, systématiquement présenté comme l'agresseur. Dans le cas de la France, la Première Guerre a entre autres su aviver la blessure de la perte de l'Alsace et de la Lorraine après la guerre de 1870-1871 (Roth 2010). Cette amputation ne constitue certes pas l'élément déclencheur de la Grande Guerre, mais elle en devient très vite, du point de vue français, un enjeu majeur, une motivation supplémentaire pour prendre une revanche sur l'ennemi héréditaire et récupérer ainsi les « provinces perdues ". II n'est dès lors pas étonnant que parmi les nombreuses chansons anciennes réactivées par la Première Guerre, on trouve en bonne place la fameuse Alsace et Lorraine, qu'on appelle communément Vous n'aurez pas l'Alsace et la Lorraine (Villemer/ Delormel 1985 : 22-23).

Un appel explicite à une guerre et à une vengeance future est clairement lancé, dont l'enjeu sera la récupération des territoires perdus. Alsace et Lorraine fait donc partie des chansons destinées à être sans cesse réactivées : enregistrée en 1899 par Henri Thomas puis par Adolphe Maréchal en 1902, elle se réincarne totalement à partir de 1914, au point de faire partie des habituelles chansons de poilus. L'enregistrement réalisé en 1919 par Jean Noté sonne quant à lui comme un chant de victoire de l'après-guerre, une promesse tenue, une prédiction réalisée... avant d'être réenregistrée en 1939 par Georges Thill et Germaine Montéro ! À l'orée de la Seconde Guerre, la chanson change une fois encore de sens et résonne comme la prédiction d'un nouveau danger imminent pour l'Alsace et la Lorraine, un appel à la résistance face

Nous nous efforçons ici de pousser l'analyse jusqu'à des chansons très récentes qui n'avaient pu être prises en compte dans des études antérieures (Hanheide/ Helms/ Glunz/ Schneider 2013). à l'ennemi. Réenregistrée en 1995 par Alain Vanzo, elle est un document mémoriel sans visée nationaliste (Klein 1989), au point qu'on peut désormais parler d'une muséification de cette chanson.

Un autre exemple est Ma p'tite Mimi dont le texte est composé en 1915 par Theodore Botrel (Ribouillaut 1996), adapté à la mélodie de la fameuse Tonkinoise de Vincent Scotto. Cette chanson réactive à son tour une mélodie plus ancienne et très connue, mais en y plaquant un texte nouveau, qui constitue un art au service de la guerre. De fait on souhaitait, en haut lieu, soutenir le moral des troupes françaises par une musique très célèbre, délibérément guillerette, et que chacun puisse facilement chanter. À l'instar de la mélodie, le texte se devait d'être léger et sémillant pour dédramatiser la guerre sur le mode systématique du décalage et de l'humour. La machine à tuer est présentée ici comme la compagne idéale du soldat qui va compenser l'éloignement de la femme aimée et devenir sa nouvelle amoureuse, thème qui rappelle Quand Madelon (mars 1914) chantée par les comiques troupiers durant la Première Guerre (Genton 2003). Le texte du refrain évoque directement le son mécanique de la mitrailleuse, qu'il va jusqu'à présenter comme une mélodie envoûtante. Et si Botrel reprend un seul vers de la chanson d'origine ( $C$ C'est comme un oiseau qui chante »), il y ajoute ironiquement un « $Z$ » pour jouer délibérément sur le registre de l'humour et du décalage :

\section{Quand ell' chante à sa manière \\ Taratata, taratata, taratatère \\ Ah que son refrain m'enchante \\ C'est comme un z-oiseau qui chante \\ Je l'appell' la Glorieuse \\ Ma p'tit' Mimi, ma p'tit' Mimi, ma mitrailleuse \\ Rosalie m' fait les doux yeux \\ Mais c'est ell' que j'aim' le mieux.}

Cette chanson (qu'on pourrait à tort interpréter aujourd'hui comme une critique morbide de la guerre sur un mode qui serait celui d'une fausse adhésion) a donné également lieu à des reprises récentes ${ }^{2}$. Là encore, il s'agit désormais de documenter cette période de conflit. Or peut-on faire mieux témoigner du passé qu'en réenregistrant des chansons qui permettent instantanément d'en retrouver les cicatrices et de faire revivre « l'esprit d'alors "?

Un troisième exemple de chanson datant du début du conflit est le Noël des enfants qui n'ont plus de maison, écrite et composée par Claude Debussy en 1915 (Reibel). La version initiale (voix et piano) va vite se transformer en un véritable chant choral de Noël. Le texte témoigne bien du nationalisme agressif qui pouvait alors toucher jusqu'aux esprits éclairés, y compris celui d'un compositeur qui, jadis, avait pourtant admiré Wagner et séjourné à Bayreuth (Becker 2013 ; Percheron 2014). Le motif de la perte se porte ici d'abord sur les cicatrices matérielles créées en France par le début

2 Entre autres Pierre Desproges en 1985 dans son spectacle du Théâtre Grévin, à l'occasion du $70^{\mathrm{e}}$ anniversaire de la bataille de Verdun. 
du conflit, les nombreuses maisons détruites dans le quart Nord-Est de la France. Pour souligner la cruauté de ces destructions, l'auteur-compositeur se place du point de vue des enfants. II souligne, par un effet de gradation, que ces pertes ne concernent pas seulement des bâtisses, mais aussi tout un système de valeurs, dont celles de l'éducation et de la religion :

\author{
Nous n'avons plus de maisons! \\ Les ennemis ont tout pris, tout pris, tout pris, \\ Jusqu'à notre petit lit ! \\ Ils ont brûlé l'école et notre maître aussi, \\ Ils ont brûlé l'église et monsieur Jésus-Christ.
}

Au-delà de leurs maisons, les enfants ont aussi perdu des êtres chers et des repères essentiels, ce qui est confirmé par la deuxième strophe qui appelle au châtiment :

[...] Noël, petit Noël, n'allez pas chez eux

N'allez plus jamais chez eux, punissez-les !

Vengez les enfants de France!

Les petits Belges, les petits Serbes, et les petits Polonais aussi !

Noël prend donc ici une saveur bien cruelle : les enfants doivent eux aussi être prêts au sacrifice et prier pour le combat et la victoire. Comme souvent dans les chants nationalistes, le mot « enfant » finit par prendre une acception métaphorique plus globale et désigner tous les Français :

Noël ! Noël ! surtout, pas de joujoux,

Tâchez de nous redonner le pain quotidien.

[...]

Noël! Écoutez-nous, nous n'avons plus de petits sabots !

Mais donnez la victoire aux enfants de France.

De fait la chanson connut le succès dès sa parution, et fut souvent chantée par des chœurs d'enfants, ce qui n'empêcha pas Claude Debussy d'être conscient du caractère larmoyant de son nationalisme, en net décalage avec la délicatesse de la musique ${ }^{3}$. Debussy sait bien qu'il a forcé le trait, et il l'exprime sans ambages dans une lettre à Paul Dukas : «Vous voyez ça d'ici : la maman est morte, Papa est à la guerre ; nous n'avons plus de petits sabots; nous aimons mieux du pain que des joujoux ; et pour conclure : 'La victoire aux enfants de France'. Ça n'est pas plus malin que ça ! Seulement, ça entre tout droit dans le cœur des citadins » (Becker 2013 ; Percheron 2014).

\section{Entre deuil et dénonciation}

L'appel à la vengeance n'est pas toujours aussi explicite que dans le texte de Debussy ; et parfois, des accents plus élégiaques dominent. Un célèbre exemple du répertoire allemand est une des nombreuses chansons réactivées en Allemagne au début de la Grande Guerre, le très ancien Ich

3 Sur les relations entre " grande musique » et Première Guerre : https://www.musik uni-osnabrueck.de/ forschung/ historische_musikwissenschaft/kompositionen_zum ersten_weltkrieg.html [consulté 5.7.2021]. hatt' einen Kameraden qui date des guerres napoléoniennes et déplore la perte d'un soldat mort au combat. La première version du texte de Ludwig Uhland Der gute Kamerad fut écrite en 1809 et mise en musique en 1825 par Friedrich Silcher. Cette chanson devint alors un classique, réadapté et réincarné à toutes les guerres, entre autres celle de 1914-18 et de 1939-1945 (Kühne 2006). Elle fait l'éloge de la camaraderie militaire, héroïse le soldat tombé au champ d'honneur, souligne la cruauté des pertes dans le camp allemand, mais sans appeler à la haine de l'ennemi.

De fait, comme d'autres chants militaires allemands, Der gute Kamerad a été partiellement entaché par son association au Troisième Reich (Oesterle 1997) et par sa reprise et ses adaptations par des personnalités néofascistes européennes telles que Jean de Brem, ce qui explique que son lien à la Première Guerre soit passé rétroactivement au second plan, laissant surtout place aujourd'hui à une version purement orchestrale, aux accents nostalgiques. Si cette chanson-hymne a été paradoxalement revendiquée par des partis très divers et diamétralement opposés, beaucoup d'entre eux ont oublié que le poème avait été rédigé lorsque le Wurtemberg était allié à Napoléon et que son intention première était plutôt celle d'un chant antiguerre.

Des chansons pacifistes vont naître à mesure que la Grande Guerre s'enlise, au sens propre comme au sens figuré. À la différence de l'art de commande des chansons " va-ten-guerre », les Antikriegslieder d'avant 1918 sont souvent anonymes, tant leurs auteurs risquaient leur vie en s'adonnant ainsi à un art hautement subversif (Verney 2014). L'exemple le plus emblématique nous est livré par la fameuse Chanson de Craonne (Marival 2005 : 549-570), composée entre 1915 et 1917. Le motif du deuil et de la séparation y devient systématique : le soldat a tout à perdre, et cette fois, ce n'est plus l'ennemi qui est pointé du doigt, mais une guerre qualifiée d'« infâme » qui ne fait que des victimes :

\section{Adieu la vie, adieu l'amour, \\ Adieu toutes les femmes \\ C'est bien fini, c'est pour toujours \\ De cette guerre infâme [...].}

Le caractère désespéré et désespérant de cette chanson anonyme est renforcé par une mélodie mélancolique, reprise de Bonsoir M'amour (1911), composée en partie sur le mode mineur et sur un rythme de valse lente. Et si le deuil programmé appelle lui aussi à une réaction de révolte, celle-ci se porte non sur l'ennemi, mais sur les décideurs ainsi que les profiteurs. Le texte prend des accents de plus en plus politiques, anticapitalistes et indignés ; et dans une langue populaire et argotique, il appelle sans ambages à la grève des soldats :

Ceux qu'ont l'pognon, ceux-là r'viendront

Car c'est pour eux qu'on crève

Mais c'est fini, car les trouffions

Vont tous se mettre en grève

Ce s'ra votre tour, messieurs les gros 
De monter sur le plateau

Car si vous voulez faire la guerre

Payez-la de votre peau [...].

Ce n'est pas un hasard si cette chanson fut notamment chantée en 1917 : elle dénonce l'usure de la guerre, les sacrifices inutiles, en particulier sur le plateau de Craonne à la suite des décisions stratégiques du général Nivelle ainsi qu'un cycle sans fin de combats violents et de permissions qui ne font que mieux ressortir la dichotomie entre l'arrière et le front. Le ton rageur annonce déjà l'esprit de certaines chansons pacifistes de l'après-guerre, notamment La butte rouge (1923) - qui sera plus tard reprise par Yves Montand et Renaud - dans laquelle Montéhus stigmatise également l'injustice sociale de la Première Guerre :

C'qu'elle en a bu du bon sang cette terre,

Sang d'ouvriers et sang de paysans,

Car les bandits qui sont cause des guerres

N'en meurent jamais, on n'tue qu'les innocents !

\section{LA GUERRE « RÉACTIVÉE » ET DÉNONCÉE EN CHANSONS}

\section{Longévité et réactivations dénonciatrices}

Entre 1917 et 1918, Bertolt Brecht écrit le texte très subversif de Die Legende vom toten Soldaten qu'il met lui-même en musique sur une mélodie simple, celle d'une valse lancinante composée sur un ton mineur (qui tranche avec le ton majeur et euphorisant de bien des chants nationalistes). Les paroles de cette chanson paraissent en 1922 en annexe du drame Trommeln in der Nacht (Brecht 1922), puis en 1927, dans le recueil Bertolt Brechts Hauspostille (Brecht 1927). Elle sera notamment immortalisée par l'interprétation de Ernst Busch" Avec l'humour noir d'un Otto Dix, le narrateur nous raconte comment, dans les derniers mois de la guerre, le cadavre d'un soldat allemand, tombé plus ou moins volontairement au champ d'honneur, est jugé déserteur et forcé de reprendre du service:

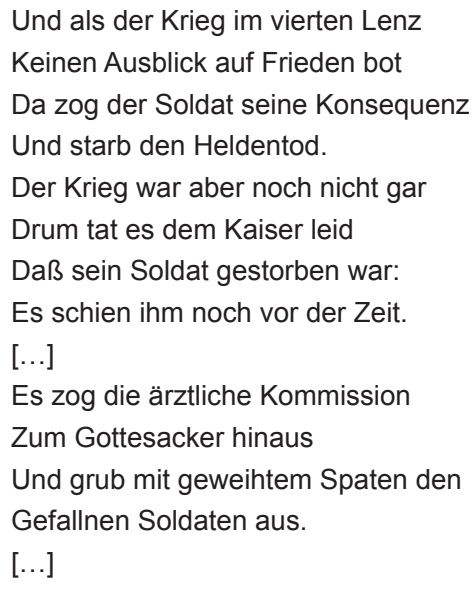

4 https://www.youtube.com/watch?v=o9pAPnKUBAM [consulté 5.7.2021].
Und der Doktor fand, der Soldat war k. v. [kriegsverwendungsfähig]

Und er drückte sich vor der Gefahr.

Und sie nahmen sogleich den Soldaten mit;

La métaphore du soldat mort, forcé de repartir au combat, illustre ironiquement la désespérance, l'arbitraire et l'absurdité des derniers mois de la Grande Guerre. L'image macabre du combattant déterré suggère l'idée que les soldats deviennent tous des morts-vivants en puissance, des marionnettes animées par un pouvoir arbitraire (de nature politico-religieuse), et « ranimées » par l'alcool, les infirmières, les prostituées, la musique militaire, le tout sur fond de propagande impériale héroïsante :

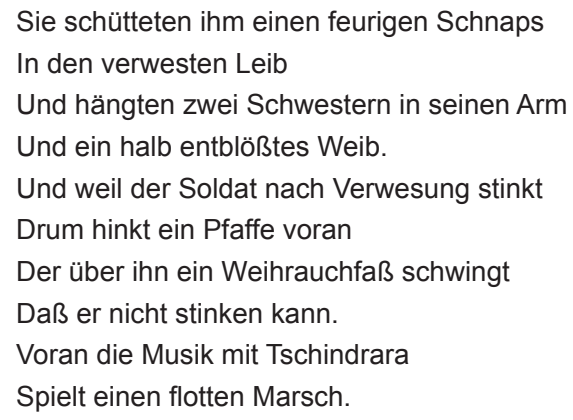

Cette chanson dénonciatrice n'est pas sans rappeler la morbidité expressionniste du jeune Gottfried Benn, ni sans annoncer Der Graben de Kurt Tucholsky, ou encore certaines descriptions crues du roman À l'Ouest rien de nouveau d'Erich Maria Remarque. Dans l'entre-deux-guerres, elle sera adaptée au chant choral par Kurt Weill en 1929, avant d'être censurée durant le Troisième Reich, tout comme le célèbre Kanonensong extrait de L'Opéra de quat' sous, créé en 1928 et paru en 1931. Or sa longévité est indéniable puisqu'elle est encore chantée aujourd'hui, étant devenue entre-temps un classique parmi les chansons pacifistes.

En comparaison, Lili Marleen dénonce la guerre d'une manière plus subtile qui va susciter des réactivations pacifistes étonnantes. Le texte, lui aussi un héritage perpétué de la Première Guerre, fut écrit en 1915 par Hans Leip, compositeur de la première mélodie de la chanson. Durant la guerre et l'entre-deux-guerres, cette chanson ne connut guère de succès. Elle ne fut redécouverte qu'à partir de 1937, grâce à deux nouvelles adaptations musicales : celle de Rudolf Zink, puis celle de Norbert Schultze qui s'imposa et fut enregistrée en 1939 par Lale Andersen (Andersen 1981). Là encore, le succès tarda à venir. Comme souvent dans l'histoire des chansons, il fut aidé par un contexte anecdotique, aux confins du mythe (Protte 2004) : plusieurs disques ayant été endommagés ou détruits lors des invasions allemandes sur le front de l'Est en 1941, on passa souvent et « faute de mieux ", sur la radio militaire allemande de Belgrade (qui était aussi très écoutée en Allemagne), cette chanson nostalgique et sentimentale. Et dès lors, civils et militaires allemands purent s'identifier à ce soldat-sentinelle amoureux d'une jeune compagne qu'il rencontre, à la nuit tombée, près d'un réverbère. C'est sans doute la conjonction de cette 
idylle amoureuse et d'une mélodie à la fois lente et nostalgique qui permit à l'alchimie d'opérer. La chanson connut dès lors un succès foudroyant dans l'Allemagne nazie et sur le front, même si plusieurs dignitaires du régime - subodorant le caractère potentiellement subversif de cette chanson - en dénoncèrent un ton qu'ils jugeaient trop macabre et démoralisant. De fait et à partir de mai 1942, la chanson fut progressivement censurée en Allemagne, notamment à cause des contacts que Lale Andersen entretenait avec des personnalités juives vivant hors d'Allemagne.

À y regarder de près, cette chanson dénonce bel et bien l'arbitraire de la machine militaire qui entrave cruellement les relations amoureuses et les rêves d'évasion:

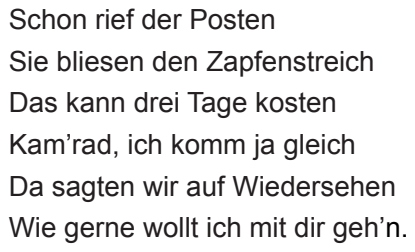

La lanterne personnifiée devient dès lors le témoin muet de cette tragédie muette : le soldat risque de mourir au combat, puis d'être remplacé par un autre homme auprès de la femme aimée. Les adversaires du régime d'Hitler et les pacifistes ne tardèrent donc pas à revendiquer cette chanson pour leur propre cause antinazie : on sait que Lili Marleen fut traduite dans de nombreuses langues et interprétée entre autres, à partir de 1943, par Marlene Dietrich devant un parterre de soldats américains. Ces réactivations subversives renforcèrent le rejet par le régime nazi d'une chanson qu'il avait pourtant commencé par porter aux nues (Rosa Sala Rose 2010). Et Lili Marleen reste aujourd'hui encore un des plus grands " tubes » planétaires allemands, pacifiste dans l'esprit, sans cesse réadapté - même en reggae ${ }^{5}$ ! Nulle provocation explicite, pourtant, dans cette chanson, à la différence des chansons pacifistes qui, comme nous allons le constater, vont fleurir après la Seconde Guerre.

\section{Provocations a posteriori}

L'une des plus célèbres chansons françaises pacifistes de l'après-guerre est sans conteste Le déserteur de Boris Vian. Sa date de composition, 1954, correspond au quarantième anniversaire du début de la Première Guerre, à la fin de la guerre d'Indochine et coïncide avec l'escalade qui allait mener au déclenchement de la Guerre d'Algérie. Sa portée devient universelle ${ }^{6}$.

Comme dans bien des chansons pacifistes, la musique que Boris Vian compose avec Harold B. Berg - est lente et nostalgique. Dans la lignée de La chanson de Craonne, le narrateur annonce sa volonté de désobéissance civique - non

\footnotetext{
5 Par exemple par Marion-Therése en 2012 : https://www.youtube.com/watch?v=Rin PV4a9r0g [consulté 5.7.2021].

6 De même Giroflée Girofla, interprétée par Yves Montand en 1955 sur son disque Chansons populaires de France (label Odéon), peut se rapporter à toutes les guerres. En comparaison, Tu n'en reviendras pas, extrait d'un poème d'Aragon adapté par Leo Ferré, se rapporte plus spécifiquement à la Première Guerre : http://www.youtube.com/watch?v=4lx9DpjdUOw [consulté 5.7.2021].
}

plus sous la forme d'une grève au cours des combats à venir, mais par une désertion réfléchie, avant même d'être mobilisé :
Monsieur le Président
Je vous fais une lettre
Que vous lirez peut-être
Si vous avez le temps
Je viens de recevoir
Mes papiers militaires
$[\ldots]$
Ma décision est prise
Je m'en vais déserter.

Le narrateur dénonce ensuite la tuerie à venir de victimes innocentes et les pertes familiales d'un passé lié à d'autres guerres - allusions simultanées à la Première et la Seconde Guerre. II invoque aussi sa propre captivité, au cours de laquelle il s'est fait dérober " [sa] femme » et " [son] âme ", renouant avec le topos de la perte de l'être aimé et des repères existentiels.

Le ton se fait ensuite de plus en plus militant : le narrateur cherchera à convaincre son prochain de ne plus participer à la guerre. II va même plus loin que La chanson de Craonne, puisqu'il invite le président en personne à donner son propre sang, plutôt que de sacrifier celui des autres:
Et je dirai aux gens :
Refusez d'obéir
Refusez de la faire
N'allez pas à la guerre
Refusez de partir
S'il faut donner son sang
Allez donner le vôtre
Vous êtes bon apôtre
Monsieur le Président [...]

La première version du Déserteur achevait même cette gradation dans l'indignation par une provocation supplémentaire, celle de la menace d'une résistance armée face aux gendarmes (Dufaud Marc 2020):

\section{Si vous me condamnez \\ Prévenez vos gendarmes \\ Que j'emporte des armes \\ Et que je sais tirer.}

Mais Mouloudji, qui fut le premier interprète de cette chanson, sut convaincre Boris Vian de changer la fin et de clore ce texte sur un ton non-violent qui fait ressortir l'arbitraire de la force armée. Plus encore qu'antimilitariste, la chanson devient pleinement pacifiste, ce qui lui valut d'être censurée dès sa parution ${ }^{7}$ :

\section{Si vous me poursuivez}

Prévenez vos gendarmes

7 Hasard troublant de l'histoire, Mouloudji interpréta la chanson pour la première fois en
public le 7 mai 1954 - le soir même de la défaite de l'armée française à Diên Biên Phu qui public le 7 mai 1954 - le soir même de la défaite de l'armée française à Diên Biên Phu qui
entraina la fin la guerre d'Indochine. 
Que je n'aurai pas d'armes

Et qu'ils pourront tirer.

Un tout autre type de provocation pacifiste a posteriori est celui de la célèbre Guerre de 14-18, de Georges Brassens, composée en 1962 - et ce n'est sans doute pas un hasard à la fin de la Guerre d'Algérie ${ }^{8}$. Sur les accents d'une musique guillerette et sur un ton humoristique, la chanson développe le thème d'une vénération affectée pour les guerres. Le narrateur établit ainsi, au fil des strophes, un palmarès des conflits marquants, à commencer par les « nobles guerres de jadis ». Mais il va parvenir inlassablement au même constat : sa guerre " préférée » est prétendument celle de 14-18... On comprend, derrière la caricature et le sarcasme, que cette première grande guerre industrielle, par ses cicatrices mémorielles, « mérite » son triste premier rang :

Depuis que l'homme écrit l'Histoire,

Depuis qu'il bataille à cœur joie

Entre mille et une guerr' notoires,

Si j'étais t'nu de faire un choix,

A l'encontre du vieil Homère,

Je déclarerais tout de suit' :

'Moi, mon colon, celle que j' préfère,

C'est la guerr' de quatorz'-dix-huit !'

La Première Guerre surpasserait celle de 1870, et même " celle de l'an quarante " (effectivement, beaucoup de Français n'avaient pas pleinement conscience, à l'époque, que la période 1945-45, à l'échelle planétaire, avait été plus meurtrière encore que la Première Guerre) :

Bien sûr, celle de l'an quarante

Ne m'a pas tout à fait déçu,

Elle fut longue et massacrante

Et je ne crache pas dessus,

Mais à mon sens, elle ne vaut guère,

Guèr' plus qu'un premier accessit,

'Moi, mon colon, celle que j' préfère,

C'est la guerr' de quatorz'-dix-huit !'

Pour finir, le narrateur prétend que la Première Guerre est celle à laquelle il rêverait de participer, suggérant au passage, comme d'autres pacifistes (entre autres Nino Ferrer dans Le Sud, enregistrée en 1975), un certain pessimisme sur la nature humaine qui ne saura sans doute s'empêcher de provoquer d'autres "grandes guerres » :

Du fond de son sac à malices,

Mars va sans doute, à l'occasion,

En sortir une - un vrai délice ! -

Qui me fera grosse impression ${ }^{9} \ldots$

8 L'expression " mon colon " témoignerait-t-elle d'une discrète allusion à la politique coloniale de la France et aux guérillas mentionnées dans la chanson ? Mais cette interprétation reste hypothétique car l'expression populaire « mon colon » était alors encore fréquente et d'abord synonyme de " mon vieux », " mon pote ».

9 Allusion probable aux nouvelles armes de destruction massive...
En attendant je persévère

A dir' que ma guerr' favorit',

'Cell', mon colon, que j' voudrais faire,

C'est la guerr' de quatorz'-dix-huit !'

Sans aller jusqu'à être censurée comme Le déserteur de Boris Vian, La guerre de 14-18 valut aussi des critiques à Georges Brassens. Dans l'émission télévisée Question de temps du 18 décembre 1978, le chanteur déclara en effet s'être vu reproché une provocation irrespectueuse visant les anciens combattants de la Première Guerre. II s'en défendit aussitôt, soulignant qu'il ironisait certes sur la Grande Guerre devenue l'archétype par excellence de la guerre en général mais non sur les combattants.

\section{Reconstitutions historiques}

À côté de l'appel explicite au pacifisme (Boris Vian) ou de I'humour implicitement provocateur (Georges Brassens), une autre particularité des chansons contemporaines consacrées pour tout ou partie à la Première Guerre consiste souvent en une reconstitution du contexte historique. À mesure que celle-ci s'est éloignée dans le temps et que les derniers témoins ont disparu, les paroliers cherchent, à partir des années 1970, à en recréer le décor et l'ambiance, ce qui permet entre autres d'atteindre le public des jeunes générations. Ce besoin d'une certaine reconstitution historique est particulièrement frappant dans Les lettres, composée par Maxime le Forestier et enregistrée en 1975. Comme dans les romans épistolaires, le narrateur s'efface pour nous livrer un document brut et apparemment "vécu " : celui d'un échange de lettres qui pourraient avoir été écrites par un soldat mobilisé dès 1912 et par sa jeune épouse. Au travers de leur long dialogue épistolaire, sur fond de musique sobre et lancinante, de Maxime le Forestier, accompagné par deux guitares et une contrebasse, l'auditeur peut être transposé dans une époque où la petite et la grande histoire sont intimement mêlées :

Août 1914, ma femme, mon amour,

En automne au plus tard, je serai de retour

Pour fêter la victoire.

Nous sommes les plus forts, coupez le blé sans moi.

La vache a fait le veau, attends que je sois là

Pour le vendre à la foire.

Le père se fait vieux, le père est fatigué.

Je couperai le bois, prends soin de sa santé.

Je vais changer d'adresse.

N'écris plus, attends-moi, ma femme, mon amour,

En automne au plus tard je serai de retour

Pour fêter la tendresse.

L'illusion liminaire d'une guerre courte et glorieuse est reconstituée très concrètement ; puis s'ensuit, par petites touches progressives, la cruelle désillusion d'une guerre qui n'en finit pas, ni pour les soldats ni pour les femmes restées seules à l'arrière. L'échange de lettres suggère discrètement 
que les femmes des poilus sont contraintes à prendre des décisions :

Hiver 1915, mon mari, mon amour,

Le temps était trop long, je suis allée au bourg

Dans la vieille charrette.

Le veau était trop vieux, alors je l'ai vendu

Et j'ai vu le vieux Jacques, et je lui ai rendu

Le reste de nos dettes.

Nous n'avons plus un sou, le père ne marche plus.

Je me débrouillerai, et je saurai de plus

En plus être économe.

Mais quand tu rentreras diriger ta maison,

Si nous n'avons plus rien, du moins nous ne devrons

Plus d'argent à personne.

À la fin de la chanson, le narrateur révèle, comme dans maint roman épistolaire, qu'il est censé avoir trouvé ces lettres dans une maison abandonnée mais que la fin brutale de l'échange épistolaire laisse présager le pire. Aux cicatrices de la guerre que constituent ces lettres-documents fictives succède la cicatrice mémorielle des monuments aux morts. Le narrateur n'ose pas en regarder les noms et interpelle l'auditeur par un simple questionnement ouvert :

Dites-moi donc pourquoi ça'est fini si tôt.

Dites-moi donc pourquoi, au village d'en haut,

Repassant en voiture,

Je n'ai pas regardé le monument aux Morts

De peur d'y retrouver, d'un ami jeune encore,

Comme la signature.

On n'est donc plus, comme chez Boris Vian, dans la morale explicite : l'interpellation se porte cette fois sur l'auditeur lui-même, sommé de s'interroger sur le sens de la Grande Guerre. Deux ans plus tard, on retrouve ce même effet de reconstitution historique et de questionnement direct dans la chanson Jaurès de Jacques Brel. Elle figure en première place de son album de 1977, comme l'un des messages les plus importants que le chanteur, retiré aux îles Marquises, aurait encore souhaité délivrer à l'approche de sa mort. Sur une mélodie dépouillée et lente, accompagnée du seul accordéon - instrument associé à la musique des tranchées -, Jaurès commence par rappeler le contexte qui précéda la guerre, mettant l'accent sur la misère des ouvriers dociles, étourdis par l'absinthe, le clergé et le patronat:

Ils étaient usés à quinze ans

Ils finissaient en débutant

Les douze mois s'appelaient décembre

Quelle vie ont eu nos grands-parents

Entre l'absinthe et les grand-messes

Ils étaient vieux avant que d'être

Quinze heures par jour le corps en laisse

Laissent au visage un teint de cendres.

Oui notre bon Maître, oui notre Monsieur [...].
Et puis la Première Guerre vient s'inviter dans le destin de ces petites gens qui se sacrifient jusqu'au bout, comme le suggère entre autre le remplacement du mot " honneur » par le terme « horreur » dans l'expression « champ d'honneur » :
Si par malheur ils survivaient
C'était pour partir à la guerre
C'était pour finir à la guerre
Aux ordres de quelques sabreurs
Qui exigeaient du bout des lèvres
Qu'ils aillent ouvrir au champ d'horreur
Leurs vingt ans qui n'avaient pu naître
Et ils mouraient à pleine peur [...].

Par cette rapide reconstitution chronologique et dramatique, le narrateur s'inscrit dans le sillage de ce qu'Anatole France avait clamé dans L'Humanité, le 18 juillet 1922 : «On croit mourir pour la patrie ; on meurt pour des industriels ». La Première Guerre serait une suite logique de la Révolution industrielle et l'exploitation des petites gens à l'usine finirait inexorablement par leur extermination sur les champs de bataille. La figure de Jean Jaurès prend donc ici tout son sens, puisqu'il s'évertua à la fois à améliorer la condition ouvrière et, surtout, à empêcher la guerre - ce qui fit de lui la victime toute désignée des bellicistes. Mais la chanson de Jacques Brel reste dans l'implicite, et comme celle de Maxime le Forestier, elle se contente de réitérer inlassablement la question du "pourquoi ?", adressée aux jeunes générations. Celles-ci sont conviées à tirer par elles-mêmes les enseignements de cette époque où la pulsion guerrière eut raison des pacifistes :

\section{Demandez-vous belle jeunesse \\ Le temps de l'ombre d'un souvenir \\ Le temps du souffle d'un soupir \\ Pourquoi ont-ils tué Jaurès? \\ Pourquoi ont-ils tué Jaurès?}

Entre les lignes se dessine un appel au devoir de mémoire, lequel constitue l'un des traits saillants des chansons les plus contemporaines.

\section{DEVOIR DE MÉMOIRE : DÉNONCIATIONS DE LA GRANDE GUERRE OU DE TOUTES LES GUERRES ?}

\section{La chanson française contemporaine et le devoir de mémoire}

Parmi les chansons françaises de la fin du $X X^{e}$ et du début du $\mathrm{XXI}^{\mathrm{e}}$ siècle consacrées à la Première Guerre, signalons L'avenir est un long passé, du groupe de rock celtique Manau. Enregistrée en 1998, cette chanson très cinématographique cherche d'abord à faire revivre à l'auditeur la mort imminente d'un soldat de 1917 : 
Près de sa tranchée, placés à 20 ou 30 mètres, la guerre des bouchers,

Nous sommes en 1917.

Tant de journées qu'il est là !

À voir tomber des âmes.

Tant de journées déjà passées sur le chemin des dames.

Marcel sent que la fin a sonné.

Au fond de sa tranchée, ses mains se sont mises à trembler.

L'odeur de la mort se fait sentir, il n'y aura pas de corps à corps,

Il sent qu'il va bientôt mourir.

La dramatisation de cette reconstitution historique « en temps réel " est renforcée par la diction dite " talk over » du chanteur, sur un rythme lancinant de rap, dont la batterie lente évoque les battements d'un cœur; et aussi par le clip vidéo ${ }^{10}$ en noir et blanc montrant des soldats dans les tranchées : la peur se lit dans les yeux du protagoniste. Puis, comme chez Maxime le Forestier et Jacques Brel, le narrateur intervient pour interpeler l'auditeur par des questions dérangeantes, avant de clore cette première strophe par la formule-phare de la chanson, elle-même éponyme du titre :

Comment un homme peut-il accepter d'aller au combat?

Et quand il sent au fond de lui qu'il ne reviendra pas.

L'homme est-il un animal ?

Comme à cette époque le mal est déjà caporal.

La main du lieutenant doucement vers le ciel s'est levée.

La suite, l'avenir est un long passé.

Ce refrain suggère que nous sommes toujours les héritiers de cette Guerre, que d'autres grands conflits ont suivi et qu'il ne saurait y avoir d'avenir sans la mémoire de ce passé destructeur. Le narrateur met ce conflit liminaire en perspective avec la Seconde Guerre - l'une des conséquences les plus funestes du traité de Versailles - et avec un autre moment dramatique, celui où un résistant s'apprête à faire sauter un train. A l'instar de Marcel pendant la Première Guerre, ce nouveau protagoniste, Jean-Marc, se résigne à trouver à son tour une mort certaine :

Le câble qu'il a placé pour faire sauter le train est bien trop court. La mort se fait sentir, mais il n'a pas de remords, comment le définir?

C'est la nature de l'homme qui l'a poussé à être comme ça.

Se sacrifier pour une idée, je crois qu'on ne résiste pas.

Comme dans Jaurès de Jacques Brel, L'avenir est un long passé soumet finalement à l'auditeur la question de la mémoire et de la résistance individuelle contre le cours de l'histoire : si nos vies sont privées de mémoire et de raison, la guerre ne risque-t-elle pas de se répéter à l'infini ? Dans le sillage de Brassens, mais cette fois avec les images dramatiques d'un vidéo-clip soulignant certains " morceaux choisis " des guerres, Manau dénonce non seulement

10 Cf. https://www.youtube.com/watch?v=GbWZodgz8_E [consulté 5.7.2021]. la Première Guerre, mais aussi, à partir d'elle, d'autres conflits passés ou potentiels, liés entre autres à une pensée d'extrême-droite :

Est-ce que tout recommence, avons-nous perdu la raison? Car j'ai vu le mal qui doucement s'installe sans aucune morale. Passer à la télé pour lui est devenu normal.

Comme à chaque fois avec un nouveau nom.

Après le nom d'Hitler, j'ai entendu le nom du front.

Et si l'avenir est un long passé, je vous demande maintenant ce que vous en pensez?

Comme Marcel et Jean-Marc ma vie est-elle tracée?

La suite, l'avenir est-il un long passé ?

Plus proche de nous dans le temps, la chanson Le soldat a été composée en 2013 sur un texte de Marie Bastide et une musique de Calogero, puis interprétée en 2014 par Florent Pagny, juste avant les commémorations du Centenaire de la Première Guerre - et l'on note, une fois de plus, l'importance des dates-anniversaires pour la composition de ces chansons. Reconstitution historique à partir des lettres fictives d'un poilu, ce sont d'abord des lettres d'amour qui cherchent à rassurer la femme aimée, à la convaincre que le conflit sera court, tout en laissant transparaitre malgré tout la dureté des tranchées et « la peur de tomber » :

\section{À l'heure où la nuit passe au milieu des tranchées \\ Ma très chère Augustine \\ Je t'écris sans tarder \\ Le froid pique et me glace et j'ai peur de tomber \\ Je ne pense qu'à toi}

Mais je suis un soldat,

Mais surtout ne t'en fais pas

Je serai bientôt là

Tu seras fière de moi

La musique entrainante et presque joyeuse de Calogero, composée sur un ton majeur, entretient l'illusion de cette assurance fragile. Mais au fil des strophes, la confiance du narrateur épistolaire s'effrite, les coups de fusil résonnent en fond sonore et le tableau devient de plus en plus réaliste et dénonciateur :

Mais je suis un soldat,

Je ne sens plus mes bras,

Tout tourne autour de moi,

Mon Dieu, sors-moi de là.

La mort et la perte du passé finissent par tout engloutir, malgré les témoignages d'amour ; et ce paradoxe fait ressortir la cruauté d'un conflit qui ne fait que des victimes de part et d'autres du front.

Les lettres chantées deviennent ainsi bel et bien des cicatrices artistiques de la Première Guerre. Mais l'aspect mémoriel de la chanson tient peut-être plus encore à son 
vidéoclip ${ }^{11}$ : en alternance avec des scènes de combat qui ne sont pas sans rappeler le film Un long dimanche de fiançailles de Jean-Pierre Jeunet (inspiré de Sébastien Japrisot, 2011), on y voit le chanteur Florent Pagny circulant tantôt dans l'ossuaire, tantôt dans le cimetière de Douaumont, où il finit par s'immobiliser devant une tombe ; puis un jeune garçon s'approche lentement de lui, auquel il va silencieusement donner la main dans un geste qui rappelle celui de François Mitterrand et Helmut Kohl (Jalabert/ Marcowitz/ Weinrich 2017) en ces mêmes lieux trente ans auparavant. Cependant nous n'avons plus à faire à deux hommes politiques, mais à un artiste qui tient la main d'un enfant, transmettant, par là-même, un devoir de mémoire à tout futur citoyen « ordinaire ».

\section{La chanson allemande contemporaine et le pacifisme « global »}

Observe-t-on cette même tendance mémorielle et dénonciatrice dans la chanson allemande contemporaine vis-à-vis de la Première Guerre ? La première réponse qui s'impose est que dans tous les langages artistiques allemands, les cicatrices de la Seconde Guerre et de la Shoah ont longtemps recouvert - on pourrait même dire occulté ! - celles de la Grande Guerre. Ce n'est donc pas un hasard si les chansons écrites après 1945 que nous avons examinées jusqu'ici ont été des exemples français. Cela ne signifie pas que la Première Guerre ait totalement disparu de la mémoire allemande, mais plutôt que les chansons pacifistes allemandes de l'après-guerre sont le plus souvent celles d'un pacifisme global : on n'évoque plus explicitement la Première Guerre mais on rejette « indifféremment » toutes les guerres passées et à venir, à l'instar, par exemple, d'un Wolf Biermann dans la chanson Soldat, Soldat enregistrée en 1965 :

\section{Soldat Soldat in grauer Norm \\ Soldat Soldat in Uniform \\ Soldat Soldat, ihr seid so viel \\ Soldat Soldat, das ist kein Spiel \\ Soldat Soldat, ich finde nicht \\ Soldat Soldat, dein Angesicht \\ Soldaten sehn sich alle gleich \\ Lebendig und als Leich.}

Comme dans le tableau Soldaten (1913) d'Emil Nolde ou La guerre de Marcel Gromaire (1923), l'uniforme et la guerre rendent anonymes, font perdre aux individus leur visage et leur identité. Le rythme de la musique, qui évoque à la fois le bruit de bottes et d'un tambour militaire, souligne le caractère abrutissant de la guerre et la répétition obsédante du terme "Soldat ». Et comme dans la chanson de Brecht, chaque soldat devient ici un cadavre en puissance.

Dans la strophe suivante, Wolf Biermann passe au mode interrogatif, puis met en garde le soldat (générique) contre

$11 \mathrm{https}: / /$ www.youtube.com/watch?v=EXKSd9WpdeU [consulté 5.7.2021]. tout futur conflit qui, dans le contexte de la course aux armements atomiques, mettrait le monde entier en échec :

\section{Soldat Soldat, wo geht das hin \\ Soldat Soldat, wo ist der Sinn \\ Soldat Soldat, im nächsten Krieg \\ Soldat Soldat, gibt es kein Sieg \\ Soldat, Soldat, die Welt ist jung \\ Soldat Soldat, so jung wie du \\ Die Welt hat einen tiefen Sprung \\ Soldat, am Rand stehst du.}

En 1981, année marquée de nombreux débats surl'opportunité du stationnement de fusées Pershing en RFA (Doppelnatobeschluss / crise des missiles), on retrouve cette même tendance globalisante dans la chanson pacifiste Frieden de l'Autrichien Georg Danzer. À ses yeux, une troisième guerre mondiale, si elle venait à se produire, surpasserait de très loin la Première et la Seconde Guerre et anéantirait quatre milliards d'êtres humains :

\author{
Aber diesmal \\ meine Herren \\ könnt's Euch sicher sein \\ daß ihr mit uns verreckt's. \\ Vier Milliarden Leben \\ vier Milliarden Tode \\ doch des is euch gleich. \\ Hört's ihr Wissenschaftler \\ ihr Politiker \\ ihr Mächtigen ${ }^{12}$
}

Und ihr baut's Raketen und Atomkraftwerke und dann Bunker - wo ihr Euch versteckt's.

Les détenteurs du pouvoir sont une nouvelle fois pris à parti : les hommes politiques, les puissants et les scientifiques qui concourent à mettre au point de nouvelles armes et centrales nucléaires... Mais plus besoin, comme chez Boris Vian, de demander à la classe politique d'aller donner son sang : la chanson suggère, sur un rythme de ballade lente, qu'une guerre technologique serait totale et, malgré les bunkers antiatomiques, n'épargnerait personne. D'où la revendication martelée d'une " paix totale » à l'échelle planétaire, le passé de la Première Guerre et des suivantes étant au fond résumé dans le seul mot « endlich » :
wir fordern jetzt von Euch:
Gebt's uns endlich Frieden
gebt's uns endlich Frieden
gebt's uns endlich Frieden für die Welt!

Au cœur de ce paysage de chansons allemandes contemporaines qui défendent un pacifisme global à l'époque de la Guerre Froide, on trouve une chanson de 1980

12 Paru sur l'album Ruhe vor dem Sturm, Polydor, 1981. 
consacrée au souvenir de la Première Guerre : Es ist an der Zeit, adaptation en allemand par Hannes Wader de No Man's Land, composée par le chanteur écossais-australien Eric Bogle. Hannes Wader reprend la figure d'un soldat tombé en Champagne en 1917 et dont la tombe n'est devenue qu'une cicatrice anonyme de la Première Guerre :

\author{
Weit in der Champagne im Mittsommergrün \\ Dort wo zwischen Grabkreuzen \\ Mohnblumen blüh'n \\ Da flüstern die Gräser und wiegen sich leicht \\ Im Wind, der sanft über das Gräberfeld streicht \\ Auf deinem Kreuz finde ich toter Soldat \\ Deinen Namen nicht, nur Ziffern und jemand hat \\ Die Zahl neunzehnhundertundsechzehn gemalt, \\ Und du warst nicht einmal neunzehn Jahre alt.
}

Dans le refrain lent, mélancolique, il reprend aussi l'idée que de telles guerres sont encore possibles aujourd'hui si les populations civiles cèdent à nouveau aux discours bellicistes des responsables politiques - rappelons que la course aux armements entre États-Unis et URSS avait atteint son paroxysme au début des années 1980 et que le stationnement des fusées Pershing en Allemagne de l'Ouest fut l'un des enjeux cruciaux de la campagne électorale de 1982 en RFA :

\section{$\mathrm{Ja}$, auch Dich haben sie schon genauso belogen \\ So wie sie es mit uns heute immer noch tun \\ Und du hast innen alles gegeben: \\ Deine Kraft, Deine Jugend, Dein Leben.}

On retrouve les questionnements sans réponse pour éveiller les consciences : ce soldat anonyme a-t-il cru jusqu'au bout à la cause officielle de la guerre ? A-t-il douté et reconnu dans la guerre elle-même son véritable ennemi ? N'a-t-il eu le temps de se rendre compte de rien ? Ou bien a-t-il, au contraire, souffert d'une lente désintégration de son corps, telle qu'ont su la dénoncer un Fernand Léger ou un Otto Dix ?

Enfin, à l'instar de Wolf Biermann et Georg Danzer, Hannes Wader clôt sa chanson par une mise en garde solennelle envers une nouvelle guerre mondiale qui, à ses yeux également, serait nécessairement la dernière. Mais plus nettement que ces deux autres chanteurs, il veut croire en la montée d'une prise de conscience pacifiste planétaire. La mémoire et les dernières cicatrices visibles de la Première Guerre peuvent précisément y contribuer :

Es blieb nur das Kreuz als die einzige Spur

Von deinem Leben, doch hör' meinen Schwur

Für den Frieden zu kämpfen und wachsam zu sein:

Fällt die Menschheit noch einmal auf Lügen herein

Dann kann es gescheh'n, dass bald niemand mehr lebt

Niemand, der die Milliarden von Toten begräbt

Doch finden sich mehr und mehr Menschen bereit

Diesen Krieg zu verhindern, es ist an der Zeit.
Aujourd'hui encore, les traces artistiques que constituent les chansons sur la Première Guerre peuvent donc être (ré)activées pour dénoncer les guerres à venir. La chanson Es ist an der Zeit est devenue un hymne pacifiste repris en chœur par des millions d'Allemands lors des manifestations berlinoises contre la deuxième guerre d'Irak, en 2003. Elle fut alors interprétée et enregistrée en trio par trois grands noms : Hannes Wader, Konstantin Wecker et Reinhard Mey ${ }^{13}$. Ces trois mêmes chanteurs l'ont reprise en 2014, au début des commémorations officielles de la Grande Guerre ${ }^{14}$. Une fois de plus, de telles cicatrices artistiques montrent leur pouvoir d'être constamment revisitées, le souvenir visant dès lors à devenir une vaccination contre toute tentation guerrière pour le présent ou pour l'avenir...

\section{CONCLUSION}

À l'issue de cette analyse d'exemples emblématiques de chansons françaises et allemandes consacrées, pour tout ou partie, à la Première Guerre, nous sommes bien loin d'avoir épuisé la question. De nombreuses autres chansons auraient mérité d'être citées et intégrées à notre réflexion. Notre choix a été de nous en tenir à un corpus limité mais représentatif de quelques grandes tendances, afin d'aborder les chansons sur la Première Guerre dans une approche de « longue durée ", de continuités et de discontinuités, d'activations et de réactivations.

Qu'elles soient bellicistes ou pacifistes, drôles ou dramatiques, la plupart de ces chansons se fondent sur le motif de la perte, de la blessure qui laissera des cicatrices et, partant, elles appellent plus ou moins explicitement à une réaction de l'auditeur. Mais là où il s'agissait encore d'encourager à punir l'ennemi diabolisé ou ridiculisé dans les chansons martiales, l'ennemi devient la guerre elle-même dans les chansons pacifistes de l'entre-deux-guerres, et, a fortiori, dans celles qui seront composées après les ravages de la Seconde Guerre. Les auteurs de chansons pacifistes, qu'ils soient allemands ou français, se retrouvent autour des thèmes de l'absurdité de la guerre, de l'hécatombe parmi les militaires et les civils, de la terreur qu'inspirent des armes de plus en plus redoutables, perverses et destructrices. Le point de vue de ces auteurs diffère en cela de l'analyse de certains historiens contemporains pour qui la période remarquablement longue de paix dans les parties industrialisées de la planète est due non pas à la revendication de la paix, mais au caractère universel (et donc dissuasif) que prendrait un nouveau conflit. À l'inverse, dans les exemples de chansons récentes, les pacifistes refusent la notion même d'armements dissuasifs. Ils estiment que ceux-ci risquent de conduire tôt ou tard à la perte de l'humanité, y compris à celle des décideurs et de ceux qui, jusque-là, avaient su tirer profit de la guerre. Leurs chansons-cicatrices revendiquent tout à la fois un rôle de témoignage mémoriel, d'alerte et de prévention.

13 Figure sur l'album Mey Wader Wecker - das Konzert, Pläne GmbH 2003

14 Cf. https://www.youtube.com/watch?v=2YkNKh6XghE [consulté 5.7.2021]. 
Nous avons constaté par ailleurs que plus la Première Guerre s'éloigne dans le temps, plus certaines chansons la reconstituent dans le détail pour mieux la faire revivre et la dénoncer. Cela dit, elles évitent généralement le piège d'une moralisation trop rigide et se limitent à des questionnements ouverts à l'adresse des jeunes générations. Les images cicatricielles parlent souvent d'elles-mêmes, soulignées par des musiques généralement lentes et minimalistes, sauf quand le parti pris est celui de l'humour noir. L'introduction de vidéoclips dans les chansons les plus récentes renforce considérablement leur dimension visuelle et cinématographique.
Mais ce constat vaut surtout pour les chansons françaises de l'après-guerre, car les Antikriegslieder allemands témoignent plutôt d'un pacifisme global, au sein duquel la Première Guerre n'apparait guère explicitement, à l'exception notable de Es ist an der Zeit. À noter d'ailleurs que cette chanson a connu un regain de faveur au début des commémorations du centenaire de la Première Guerre mondiale, ce qui confirme le constat de la fécondité artistique des dates-anniversaires de la Grande Guerre, celles où les cicatrices du conflit ne demandent qu'à être réveillées, du plus profond de notre mémoire collective et individuelle.

\section{Bibliographie}

Andersen, Lale (1981), Leben mit einem Lied, München, Dtv.

Becker, Annette (2013), « Debussy en Grande Guerre », in Chimènes, Myriam/ Laederich, Alexandra (éd.), Regards sur Debussy, Paris, Fayard.

Botrel, Théodore (1915), Les Chants du bivouac. $1^{\text {er }}$ août - 31 décembre 1914. Refrains de guerre. Paris, Payot.

Brecht, Bertolt (1922), Trommeln in der Nacht, München, Drei Masken.

Brecht, Bertolt (1927), Bertolt Brechts Hauspostille, Berlin, Propyläen. Dufaud, Marc (2020), J'irai chanter sur vos tombes, Lille, éd. invenit.

Genton, François (2003-2004), « Quand Madelon et Lili Marleen : ce que disent les chansons ", in Chroniques allemandes, $\mathrm{n}^{\circ} 10$, 97-108.

Hanheide, Stefan/ Helms, Dietrich/ Glunz, Claudia/ Schneider, Thomas F. (éd.) (2013), Musik bezieht Stellung - Funktionalisierungen der Musik im Ersten Weltkrieg, Osnabrück, Universitätsverlag, V\&R unipress.

Jalabert, Laurent/ Marcowitz Reiner/ Weinrich Arndt (éd.) (2017), La longue mémoire de la Grande Guerre. Regards croisés francoallemands de 1918 à nos jours, Villeneuve d'Ascq, Septentrion.

Japrisot, Sébastien (2011), Un long dimanche de fiançailles, Paris, Denoël.

Klein, Jean-Claude (1989), Florilège de la chanson française, Paris, Bordas.

Kühne, Thomas (2006), Kameradschaft. Die Soldaten des nationalsozialistischen Krieges und das 20. Jahrhundert, Göttingen, Vandenhoeck \& Ruprecht.

Lied und populäre Kultur. Zeitschrift des Wissenschaftsverlags Waxmann. Volksliedarchiv Freiburg im Breisgau.

Marival, Guy (2005), « La Chanson de Craonne. De la chanson palimpseste à la chanson manifeste » in Offenstadt, Nicolas (éd.), Le Chemin des Dames de l'événement à la mémoire, Paris, Stock.

Oesterle, Kurt (15-11-1997), « Die heimliche deutsche Hymne », in Schwäbisches Tagblatt, Tübingen.

Percheron, Bénédicte (2014), « 'Claude Debussy, musicien français' : Les années de guerre d'un compositeur », in Les Rendez-vous de 14-18, ESPE de Rouen [https://hal.archives-ouvertes.fr/hal-01403762].

Protte, Katja (2004), « Mythos ,Lili Marleen“ - Ein Lied im Zeitalter der Weltkriege », in Militärgeschichtliche Zeitschrift (18-4-2014), 63.

Reibel, Emmanuel, Les Compositeurs et les Musiciens pendant la Grande Guerre: https://www.youtube.com/watch?v=dV3Sp18GTA0

Ribouillault, Claude (1996), La musique au Fusil, Rodez, éd. du Rouergue.
Rose, Rosa Sala (2010), Lili Marleen. Die Geschichte eines Liedes von Liebe und Tod, München, dtv (trad. en allemand par Andreas Löhrer).

Roth, François (2010), Alsace-Lorraine. Histoire d'un « pays perdu » de 1870 à nos jours, Nancy, éd. de la Place Stanislas.

Saka, Pierre/ Saka, Jean-Pierre (2004), L'histoire de France en chansons, Paris, Larousse.

Verney, Jean-Pierre (2014), Chansons contre la guerre - Antiwar songs - Antikriegslieder, Paris, Casterman (ill. Jacques Tardi).

Vian, Boris (2003), CEuvres, tome 11, Paris, Fayard.

Villemer, Gaston/ Delormel, Lucien (1985), Les Chansons d'Alsace-Lorraine, Paris, Barthlot / Marpon \& Flammarion.

\section{Chansons citées}

Alsace et Lorraine (1871) (texte : Gaston Villemer/ Hippolyte Nazet musique : Ben Tayoux).

Ma p'tite Mimi (1915) (texte : Theodore Botrel - musique : Vincent Scotto). Noël des enfants qui n'ont plus de maison (1915) (texte et musique : Claude Debussy).

Ich hatte einen Kameraden (texte 1809, musique 1825) (texte : Ludwig Uhland - musique : Friedrich Silcher).

Chanson de Craonne (texte entre 1915 et 1917/ musique 1911) (texte anonyme - musique : Adelmar Sablon).

Bonsoir M'amour (1911) (texte : Raooul Le Peltier - musique : Adelmar Sablon).

La butte rouge (1923) (texte : Montéhus - musique : Georges Krier). Die Legende vom toten Soldaten (entre 1917 et 1918) (texte et musique : Bertolt Brecht).

Kanonensong (1928) (texte : Bertolt Brecht - musique : Kurt Weill). Le déserteur (1954) (texte et musique : Boris Vian).

Giroflée Girofla (1935) (texte : Rosa Holt - musique : Henri Goublier) interprétée par Yves Montand en 1955.

Tu n'en reviendras pas (1961) (texte adapté d'un poème de Louis Aragon - musique : Leo Ferré).

La guerre de 14-18 (1962) (texte et musique : Georges Brassens). Les lettres (1975) (texte et musique: Maxime le Forestier).

Jaurès (1977) (texte et musique : Jacques Brel).

L'avenir est un long passé (1998) (texte et musique : groupe Manau : Martial Tricoche, Cédric Soubiron, Hervé Lardic).

Le soldat (2013) (texte : Marie Bastide - musique : Calogero - interprète : Florent Pagny).

Soldat, Soldat (1965) (texte et musique : Wolf Biermann).

Frieden (1981) (texte et musique : Georg Danzer).

Es ist an der Zeit (1980) (texte : Hannes Wader - musique : Eric Bogle). 